Bryn Mawr College

Scholarship, Research, and Creative Work at Bryn Mawr College

Graduate School of Social Work and Social

Graduate School of Social Work and Social

Research Faculty Research and Scholarship

Research

2012

\title{
Means, Intent, Lethality, Behaviors, and Psychiatric Diagnosis in Latina Adolescent Suicide Attempters
}

Carolina Hausmann-Stabile

Bryn Mawr College, chausmanns@brynmawr.edu

Jill A. Kuhlberg

Luis H. Zayas

Allyson P. Nolle

Stephanie L. Cintron

Let us know how access to this document benefits you.

Follow this and additional works at: http://repository.brynmawr.edu/gsswsr_pubs

Part of the Social Work Commons

\section{Custom Citation}

Hausmann-Stabile, C. J.A. Kuhlberg, L.H. Zayas, A.P. Nolle, S.L. Cintron. 2012. Means, Intent, Lethality, Behaviors, and Psychiatric Diagnosis in Latina Adolescent Suicide Attempters. Professional Psychology: Research and Practice 43.3: 241-248.

This paper is posted at Scholarship, Research, and Creative Work at Bryn Mawr College. http://repository.brynmawr.edu/gsswsr_pubs/73

For more information, please contact repository@brynmawr.edu. 


\title{
Means, Intent, Lethality, Behaviors, and Psychiatric Diagnosis in Latina Adolescent Suicide Attempters
}

\author{
Carolina Hausmann-Stabile and Jill A. Kuhlberg \\ Washington University in St. Louis
}

Allyson P. Nolle

Washington University in St. Louis

\author{
Luis H. Zayas \\ The University of Texas at Austin
}

Stephanie L. Cintron
Pace University

\begin{abstract}
This article describes the means, intent, lethality, behavioral profiles, and psychiatric diagnoses of adolescent Latina suicide attempters. From a large, mixed-method project studying the sociocultural processes of Latina suicide attempts, we selected 76 participants for this report. In addition to quantitative research data, medical records were available for all 76 participants, as was qualitative data from in-depth interviews for 34 of them. Using the qualitative and quantitative research data, we explored intent and behavioral profiles of the suicidal adolescents. Medical records provided additional information about the means the adolescents used in their attempts, and about their psychiatric diagnoses. The lethality of suicide attempts was coded using the Lethality of Suicide Attempt Rating Scale (LSARS) and the Lethality of Suicide Attempt Rating Scale-Updated (LSARS-II). Findings showed that Latina adolescent suicide attempts are low in lethality. Consistent with the literature, most adolescents reported that they attempted by using means available in their homes (cutting and overdosing with medications were the predominant methods). Interesting discrepancies emerged when comparing adolescents' self-reported behavioral profiles with clinicians' psychiatric diagnoses. This report has implications for diagnosis and treatment approaches for both inpatient and outpatient service providers.
\end{abstract}

Keywords: Latina adolescent, suicide attempt, intent, lethality, means, behavioral profile, psychiatric diagnosis

Latino adolescents, which account for $41.1 \%$ of the youth in the United States (Pew Hispanic Center, 2009), are at great risk for behavioral and psychiatric disorders (Centers for Disease Control and Prevention, 2010). More specifically, Latina teens are at high risk for suicidal behaviors (Garcia, Skay, Sieving, Naughton, \& Bearinger, 2008; CDC, 2010), and are almost twice as likely as
non-Hispanic teenage girls to make suicide attempts that require medical attention (CDC, 2010).

Several studies have discussed the reasons behind this phenomenon (Turner, Kaplan, Zayas, \& Ross, 2002; Zayas, Lester, Cabassa, \& Fortuna, 2005), the characteristics of Latina suicidal attempts (Zayas, Gulbas, Fedoravicius, \& Cabassa, 2010), and the
Editor's Note. This is one of 13 accepted articles received in response to an open call for submissions on Multicultural Practice in Professional Psychology.-MCR

CAROLINA HAUSMANN-STABILE obtained her master's degree in psychology from the Universidad Nacional de Cordoba, Argentina, and a master's degree in social work at Fordham University in New York. She is currently pursuing her PhD in social work at the Warren Brown School of Social Work, Washington University in St. Louis. Ms. Hausmann-Stabile's areas of research interest are centered in Latino child and adolescent mental health, and the dynamics of family change processes resulting from social crisis and immigration.

JILL A. KUHLBERG is currently a doctoral student at the George Warren Brown School of Social Work at Washington University in St. Louis, where she received her master's in social work in 2009. Her research interests are in the social determinants of health of children in Latino communities.

LuIS H. ZaYAS is the Dean of the School of Social Work at The University of Texas at Austin. Dr. Zayas received his bachelor's degree from Manhattan College, a master's in social work degree from Columbia University, and a PhD in developmental psychology, also from Columbia University. His research interests are centered in Hispanic mental health and adolescent development.
ALLYSON P. NOLLE received her master's degree in social work at the George Warren Brown School of Social Work in 2009, where she completed a graduate research fellowship with the Center for Latino Family Research. She currently practices social work in St. Louis, Missouri. StePHANIE L. CinTRON is currently a doctoral student in the PsyD Program in School-Clinical Child Psychology at Pace University. She received her master of science in education in school psychology with a bilingual specialization in 2011. She is currently a student affiliate of the American Psychological Association and the New York State Psychological Association. Her research interests are in cultural identity and its relationship to psychopathology.

We thank Melissa Jonson-Reid, $\mathrm{PhD}$ and Andrea Campetella, $\mathrm{PhD}$ for their insightful comments and suggestions.

Data collection and manuscript preparation was provided by a Research Grant from the National Institute of Mental Health to Luis H. Zayas, PhD (NIMH grant R01 MH070689), and by a Training Grant from the National Institute of Mental Health of the U.S. Public Health Service (NIMH-T32 MH19960).

CORRESPONDENCE CONCERNING THIS ARTICLE should be sent to Carolina Hausmann-Stabile, George Warren Brown School of Social Work, Washington University, CB 1196, One Brookings Drive, Saint Louis, MO 63130-4899. E-mail: chausmann@gwbmail.wustl.edu 
challenges encountered when providing professional care to these patients (Spirito \& Overholser, 2003). In this report, we analyze the means, lethality, and intent of the girls' actions, and compare the adolescents' psychiatric diagnoses to the girls' own description of their behavioral profiles.

\section{Suicide Attempts and Means}

The method used to attempt suicide can have a determining role in its outcome (Overholser \& Spirito, 2003). Adolescents using highly lethal methods (e.g., self-inflicted gun shots) report the strongest desire to end their lives, as compared to adolescents using less lethal methods (e.g., superficial self cutting; Nasser \& Overholser, 1999). Drug overdoses are the most common attempt methods among adolescents (Spirito, Overholser, \& Stark, 1989). Most adolescent females attempt suicide by using means that are available in their homes while other people are around (Garfinkel, Froese, \& Hood, 1982).

\section{Suicide Attempts and Intent to Die}

The Institute of Medicine defines a suicide attempt as a nonfatal, self-inflicted destructive act with the explicit or implied intent to die (Goldsmith, Pellmar, Kleinman, \& Bunney, 2002). Intent refers to the desire to end one's life and includes the person's knowledge of the risk and the means to achieve the desired outcome (Silverman, Berman, Sandal, O'Carroll, \& Joiner, 2007). The vexing issue is determining intent in the absence of an explicit statement by the person, or in the face of a person's retraction after the attempt (Silverman et al., 2007). Adolescents often recant their statements of attempting suicide to avoid hospitalization, or sometimes give health care providers discrepant accounts of their behaviors (Velting, Rathus, \& Asnis, 1998). In addition, they may engage in self-injurious behavior that deliberately inflicts some destruction or alteration to the body tissue (e.g., cutting) without expressing or having suicidal intent (Muehlenkamp \& Gutierrez, 2004). When intent is ambiguous, mental health professionals are less likely to agree on whether or not an attempt was made (Wagner, Wong, \& Jobes, 2002).

\section{Suicide Intent and Lethality}

Lethality is the inherent danger and the potential for death associated with the suicidal act (Berman, Shepard, \& Silverman, 2003). A minimal association between the degree of suicide intent and the extent of medical lethality has been found, indicating that suicide intent and lethality are independent dimensions of suicideattempt behavior (Brown, Henriques, Sosdjan, \& Beck, 2004). Among adolescents, the lethality of a suicide attempt may be determined less by their intent to die than by their access to lethal methods (Spirito \& Overholser, 2003). Furthermore, adolescents often have inaccurate perceptions of the risks associated with an attempt (Harris \& Myers, 1997). For mental health professionals, low lethality attempts are often hard to distinguish from self-injury behaviors (Wagner et al., 2002).

\section{Psychiatric Diagnosis and Suicide Attempts}

Several studies have investigated the relationship between psychopathology and suicidal behavior. In a school-based sample, adolescents who engaged in suicidal behaviors showed higher severity of psychopathology than their nonsuicidal peers (Mazza \& Reynolds, 2001). Major depressive disorder is the most common psychopathology diagnosed among adolescents, and it is associated with the greatest risk of suicide attempts (Jacobson, Muehlenkamp, Miller, \& Turner, 2008). Young females who have attempted suicide and have been diagnosed with severe personality disorder have been found to be at risk for repeat attempts (BlascoFontecilla et al., 2009). However, the severity of a personality disorder was not associated with the lethality of the attempt (Blasco-Fontecilla et al., 2009). The literature has stressed the role played by impulse-control issues among low-lethality female suicide attempters (Baca-Garcia et al., 2005).

\section{Method}

We used three nested data sets for this project: research questionnaires, qualitative interviews, and a retrospective chart review based on medical records. We selected 76 participants from a large, mixed-method project studying the sociocultural processes of Latina suicide attempts. These 76 participants were chosen because, in addition to data from the research questionnaires, their medical records were available and could be used to develop retrospective chart reviews. For 34 of the 76 participants, there were also qualitative data available from in-depth interviews.

\section{Participants}

Participants were self-identified Latinas between the ages of 11 and 19 from the New York City metropolitan area. In the preceding six months to the meeting with the research team, participants had reported behaviors that were labeled as suicidal by a mental health professional. The labeling of these behaviors took place in clinical settings (e.g., emergency pediatric rooms) to which the adolescents were referred or walked in on their own to receive medical attention as a result of their suicidal behaviors. Participants were recruited from a large social service and mental health agency and from the psychiatric outpatient, inpatient, and emergency services of several hospitals. All participants were receiving mental health services at the time of their enrollment. The adolescents were referred to the study by their treating clinician, who assessed that the girls were medically and psychiatrically stable enough to participate in the project - that is, to undergo the duration and characteristics of the research interview. Participants were thus not randomly selected; they were adolescents receiving services at the recruitment sites. Referring clinicians were trained in the eligibility criteria for the project. The adolescents and their parents provided written assent and consent for their voluntary participation in the study. Prospective participants who were outside the age range, were in foster care at the time of the suicide attempt, or had a diagnosis of mental retardation or psychotic disorder were excluded from the project. The exclusion of patients with psychotic disorders was done in an effort to protect these subjects and to avoid collecting data presenting confounding psychiatric issues that were beyond the scope of a socioculturalprocesses research project. Trained, master's-degree-level social workers or doctoral-level psychologists, all bilingual Latinas, conducted the interviews. Interviews typically required 50 minutes. All girls were paid for their participation. The Human Research 
Protections Office of Washington University in St. Louis, and each of the participating agencies and hospitals approved the procedures used in the project.

\section{Data Sources}

For this report we drew data from the questionnaires completed by 76 adolescents, the medical records of the same 76 adolescents, and the in-depth interviews conducted with 34 of them. From the questionnaires, we drew demographic and behavioral data, and the number of previous attempts, if any. From the medical records, we developed a retrospective chart review with information about the psychiatric diagnosis given to each adolescent after her most recent suicide attempt, and about the means, injury extent, and circumstances of the attempt. The chart review was developed using the Lethality of Suicide Attempt Rating Scale (LSARS) (Smith, Conroy \& Ehler, 1984) and the Lethality of Suicide Attempt Rating Scale-Updated (LSARS II) (Berman et al., 2003) models. Finally, from the in-depth interviews we drew qualitative data that allowed us to explore the intent to attempt suicide from each girl's perspective. All interviews were analyzed using a structured coding, and a statement of intent was compiled for each one.

\section{Variables}

Means. The manner in which the adolescent attempted suicide was collected from the medical charts. This variable included actions (e.g., facing traffic, ingesting pills) and devices (e.g., kitchen knife). For cases in which the attempt was made by ingesting substances, doses (e.g., Tylenol doses) as well as subject's body weight were recorded.

Lethality of the attempt. The LSARS (Smith et al., 1984) describes the likelihood of death as a result of a suicide action. The scale is supplemented by the LSARS-II( Berman et al., 2003), which offers a complementary appendix listing drugs, chemicals, and lethal ranges of ingestion by body weight. The instrument is an 11 -point scale $(0=$ death is an impossible result to $10=$ death is almost a certainty) that reflects the severity of the suicide attempt. Suicidal behavior scoring above scale-point 3.5 would be labeled as serious by most clinicians (Range \& Knott, 1997). The strengths of this instrument include that the following: (a) it can be used by nonmedical raters, (b) it can be applied to historical data, and (c) it can help clinicians to rank the lethality of the attempt in an unbiased way (Smith et al., 1984). Interrater reliability for the instrument has been found to be high, from $r=.81$ for social workers to $r=.88$ for psychologists (Smith et al., 1984; Nasser \& Overholser, 1999). For our study, two trained researchers working independently scored attempt-lethality using the information collected from the participants' medical charts. Interrater reliability was perfect $(r=1.00)$.

Psychiatric diagnosis. From the medical records, we collected the first psychiatric diagnosis given to the adolescents following their suicide attempts. We reported the diagnosis using the The Diagnostic and Statistical Manual of Mental Disorders, 4th edition text revision (DSM-IV TR) multiaxial method (American Psychiatric Association, 2000). At times, clinicians gave the adolescents more than one diagnosis on each axis. Axis I diagnoses were collapsed by the research team into mood, adjustment, anx- iety, and impulse-control disorder categories. This clustering is based upon the DSM-IV-TR Axis I diagnosis classification (APA, 2000). Axis II diagnoses, which comprise personality disorders, were clustered into borderline, mood, and deferred categories. Axis III diagnoses were collapsed into three categories: asthma, other (e.g., obesity), and none. Axis IV psychosocial and environmental stressors were collapsed into four categories: family stressors, history of abuse, peer problems, and other (i.e., relocation, absence of parent, financial problems). Axis V Global Assessment of Functioning (GAF) scores were collapsed into 10-point ranges (i.e., 21-30, 31-40).

Behavioral profile: Internalizing and externalizing behaviors. The adolescents' self-reported internalizing and externalizing behavior factors were drawn from the subscales of the same names, which came from the Youth Self-Report (YSR) (Achenbach, 1991), one of the research questionnaires used in this study. Research has established the YSR reliability and validity for samples of English as well as Spanish speakers (Achenbach, 1991). The YSR internalizing and externalizing behavior subscales consist of 32 and 20 items, respectively, rating specific social, behavioral, and emotional problems using a 3-point Likert scale (not true to very true or very often). The internalizing behavior subscale includes withdrawn depressive behaviors, anxious depressive behaviors, and somatic symptoms. For this study, the subscale's coefficient alpha was .91. The externalizing behavior subscale includes aggressive and rule-breaking behaviors. For this study, the externalizing behavior subscale coefficient alpha was .91. We report the raw scores from both scales and subscales (for the internalizing behaviors: withdrawn depressive, anxious depressive, and somatic symptoms subscales; for the externalizing behaviors: aggressive and rule-breaking behavior subscales), as well as the percentages of participants that reached borderline-clinical or clinical range in the subscales, using the $t$ score cutoffs recommended in the YSR manual (Achenbach, 1991).

Attempt recorders. The mental health professionals who recorded the information in the medical charts, and who labeled the adolescents' behaviors as suicidal, were coded once for their profession (i.e., nurse, psychiatrist, social worker/psychologist) and again for their workplace setting (i.e., emergency room, outpatient clinic).

Intent to die. The adolescent's intention to die at the time of the suicide attempt was drawn from 34 subjects who completed qualitative interviews, and coded as "yes," "no," or "unsure." "Unsure" indicates that the adolescent was uncertain about her intention.

Lifetime attempts. The number of attempts reported by the adolescents, including the most recent, was collected from the research questionnaires.

Covariates. From the research questionnaires we recorded covariates including the age of the adolescent, whether or not she was U.S.-born, and the self-identified Hispanic cultural group (e.g., Mexican) (see Table 1).

\section{Analysis Techniques}

The data were analyzed using SAS statistical software version 9.3 (SAS Institute, Cary, NC). Univariate descriptive statistics were performed on all the numerical variables studied and for all 76 participants included in this report. Qualitative data analyses 
Table 1

Data Sources and Variables

\begin{tabular}{|c|c|c|}
\hline Mixed-method study & & Retrospective chart review \\
\hline $\begin{array}{l}\text { Structured questionnaire }(N=76) \\
\text { - Covariates: Age, country of origin, respondent's } \\
\text { self-identified Hispanic cultural group) } \\
\text { - Lifetime attempts } \\
\text { - Behavioral profile: Internalizing and externalizing } \\
\text { behaviors (YSR, Acherbach, 1991) }\end{array}$ & $\begin{array}{l}\text { Qualitative interview }(n=34) \\
\text { - Intent to die }\end{array}$ & $\begin{array}{l}\text { Medical charts }(N=76) \\
\text { - Psychiatric diagnosis } \\
\text { - Attempt version recorders } \\
\text { - Settings of care } \\
\text { - Means of the suicidal action } \\
\text { - Context of the suicidal action } \\
\text { - Attempt lethality: LSARS (Smith et al., 1984) and } \\
\quad \text { LSARS II (Berman et al., 2003) }\end{array}$ \\
\hline
\end{tabular}

were performed on the in-depth interviews, which were available for 34 of the 76 participants. All interviews were audio-recorded to increase their descriptive validity (Maxwell, 1992). Interview transcripts were analyzed using a structured coding scheme that identified text segments that described the intent of their suicidal behaviors. A statement of intent was compiled for each transcript.

\section{Results}

\section{Demographics}

The 76 girls whose data inform this paper were on average 15.5 years old $(S D=1.8)$ and in ninth grade. The majority of the participants were born in the US $(76.3 \%)$, and were of Puerto Rican $(42.1 \%)$, Dominican (31.6\%) or Mexican (18.4\%) descent (see Table 2).

\section{Means}

The most common method for attempting suicide was cutting using an array of objects, such as knives, pieces of glass, and nail files $(42.1 \%)$; and overdosing with over-the-counter or prescribed medications $(36.8 \%)$. Some attempters combined these two methods $(9.2 \%)$. It is important to note that mental health professionals labeled the self-cutting of all the adolescents in this report as suicidal. Less common attempting methods were jumping off buildings $(7.9 \%)$, suffocating by placing plastic bags over their heads, and trying to hang themselves (3.9\%).

\section{Lethality}

Lethality scores ranged from $0(6.7 \%)$, in which death is an impossible result of the "suicidal" behavior, to 5 (2.7\%), in which

Table 2

Demographics of Latina Adolescents and Their Suicide Attempts $(\mathrm{n}=76)$

\begin{tabular}{lcc}
\hline & Mean $(S D)$ & Frequency (\%) \\
\hline Age at interview & $15.5(1.8)$ & \\
US Born & & $58(76.3 \%)$ \\
Cultural Group & & $32(42.1 \%)$ \\
Puerto Rican & & $24(31.6 \%)$ \\
Dominican & $12(18.4 \%)$ \\
Mexican & $8(7.9 \%)$ \\
Other & \\
\hline
\end{tabular}

the chosen method has an equivocal outcome (10 being the highest point of the scale). The majority of participants had low lethality scores, or below 3.5 (84\%). Twelve participants, however, had lethality scores higher than $3.5(16 \%)$, which can be labeled as serious (Range \& Knott, 1997). The majority of the low-lethality attempters had a score of $1(61.3 \%)$, for which a lethal outcome is very highly improbable. They were followed by those scoring 2 (16\%), for whom death is improbable as an outcome of the act. In the higher lethality group, the suicidal actions of 10 participants scored 3.5 (13.1\%), meaning that death is improbable so long as first aid is administered by the victim or other agent. Finally, only two participants were coded with a lethality score of $5(2.7 \%)$.

\section{Psychiatric Diagnoses}

Axis I. Psychiatric diagnoses given by mental health professionals to the Latina adolescents following their latest suicide attempt report were collapsed into four categories: mood, adjustment, anxiety, and impulse control disorders. The majority of the adolescents were given one Axis I diagnosis (64\%), about a quarter received two $(26.5 \%)$, and only a tenth of the sample received three or more Axis I diagnoses (9.5\%). Following their suicide attempts, almost two thirds of the adolescents were given a mood disorder diagnosis $(62.7 \%)$. The most prevalent mood disorder diagnosis was depressive disorder (48\%), followed by other mood disorders (i.e., mood disorder not otherwise specified; $13.3 \%$ ), and dysthymia (8\%). One third of the adolescents were diagnosed with an adjustment disorder $(30.7 \%)$. Adjustment disorder with depressed mood was the most common label used within this diagnosis category $(21.1 \%)$. Clinicians diagnosed 11 suicide attempters with anxiety disorders $(14.7 \%)$. Posttraumatic stress disorder was the most common anxiety disorder diagnosis given to

Table 3

Latina Adolescent Self-Reports on Internalizing and Externalizing Behaviors

\begin{tabular}{lcc}
\hline & Raw Score $(S D)$ & Borderline or Clinical \\
\hline Internalizing $(n=73)$ & $26.0(11.3)$ & $51(69.9 \%)$ \\
Withdrawn & $7.3(2.9)$ & $28(37.3 \%)$ \\
depressive & & \\
Anxious depression & $10.7(5.7)$ & $12(16.4 \%)$ \\
Somatic symptoms & $8(4.2)$ & $34(45.3 \%)$ \\
Externalizing $(n=73)$ & $18.6(10.4)$ & $40(54.8 \%)$ \\
Aggressive & $11.9(6.5)$ & $32(45.7 \%)$ \\
Rule Breaking & $6.7(4.9)$ & $32.8 \%)$ \\
\hline
\end{tabular}


the teens $(9.3 \%)$. Only a small group of adolescents was given an impulse control disorder (4\%) or oppositional defiant disorder (4\%) diagnosis.

Axis II. Clinicians diagnosed only a small number of girls with personality disorders (4\%). These youngsters were labeled as having borderline personality traits.

Axis III. The majority of records (57.9\%) did not report any medical conditions for the adolescents included in this study. The most prevalent physical problem was asthma (18.4\%).

Axis IV. The majority of adolescents was given an Axis IV diagnosis $(77.6 \%)$. The most prevalent diagnoses were related to family relationship stressors (58\%), followed by a history of abuse (17\%). Among those participants with a history of abuse, the majority had been the victims of sexual abuse (60\%) while some were physically abused (40\%).

Axis V. Scores were collapsed into suggested 10-point ranges (21-30, 31-40, 41-50, 51-60). The average Global Assessment of Functioning (GAF) score was $52.5(S D=5.8)$, and ranged from 30 to 60 . The majority of adolescents were ranked between GAFs of 50 and $60(83.9 \%) ; 22$ adolescents scored $55(19 \%)$.

\section{Behavioral Profiles: Internalizing and Externalizing}

The adolescents reported their internalizing and externalizing behaviors by completing a questionnaire with the YSR subscales (Achenbach, 1991; see Table 2). The average raw score within the internalizing subscale was $26(S D=11.3)$. More than two thirds of the participants scored in this subscale within the borderlineclinical or clinical range (69.9\%). This subscale is composed of three subscales: withdrawn depressive behaviors, anxious depressive behaviors, and somatic complaints. The average raw score within the withdrawn depressive behaviors subscale was 7.3 $(S D=2.9)$. Over one third of the study participants scored within this scale's borderline-clinical or clinical range (37.3\%). In the anxious depressive behaviors subscale, the adolescents scored on average $10.7(S D=5.7)$, and 12 participants scored within the borderline-clinical or clinical range $(16.4 \%)$. Somatic complaints subscale scores were on average $8(S D=4.2)$, with almost half of the sample scoring within the borderline-clinical or clinical range $(45.3 \%)$.

The externalizing behaviors subscale from the YSR was used for adolescents to report their own associated behaviors (Achenbach, 1991). On average, the adolescents scored 18.6 in this subscale $(S D=10.4)$. More than half of the adolescents were in the borderline-clinical or clinical range in this subscale $(54.8 \%)$. The YSR externalizing behaviors subscale is built on two subscales inquiring about aggressive behaviors and rule-breaking behaviors. When reporting about their aggressive behaviors, the adolescents scored on average 11.9 ( $S D=6.5$ ), with $25.7 \%$ reaching the borderline-clinical or clinical range. Rule-breaking scores were high, with an average of $6.7(S D=4.9)$. Forty-three percent $(43.8 \%)$ of the sample reached the borderline-clinical or clinical range when reporting about their rule breaking behaviors.

\section{Intent}

Intent was determined only for the participants completing the in-depth qualitative interviews $(n=34)$. When asked about the suicide attempt objective, slightly more than half of this subsample
(53\%) said that they intended to die. As stated by one participant, "I threatened to kill myself. Said I was going to kill myself, you're not gonna see me no more, I'm gonna die." Six participants (18\%) shared that they did not seek to die because of their suicidal actions. "I was like, I didn't, I didn't even want to ki-what are you talking about? I was not trying to attempt suicide." These adolescents explained that through their actions they tried to alleviate an internal state of discomfort: "I just wanted relief of something." Finally, 10 participants (28\%) were unsure of their intent: "For me, I don't know, like ... I wasn't really thinking what was gonna happen. I just did it."

\section{Attempt Recorders, Settings of Care}

Following their suicide attempts, the Latina teens in this study first contacted the following health care providers: (a) social workers $(65.2 \%)$, (b) psychiatrists $(33 \%)$, (c) others (i.e., phone intake staff, $7.6 \%$ ), (d) nurses (3\%), and (e) general physicians $(2.3 \%)$. Also after their suicide attempts, the adolescents received care in emergency rooms (56.4\%), outpatient mental health clinics $(25.8 \%)$, psychiatric inpatient units $(11.7 \%)$, and other health care settings $(5.8 \%)$.

\section{Lifetime Attempts}

One third of adolescents reported that the attempt for which they were enrolled into the study was their first one $(32.8 \%)$. The other two thirds, however, had made previous suicide attempts $(61.7 \%)$. A few participants declined to provide information about previous suicide attempts $(5.5 \%)$. Some had a lifetime suicide history of two attempts $(28.9 \%)$, while others had attempted between three and five times $(30.2 \%)$. A few had a lifetime history of more than six attempts $(2.6 \%)$.

\section{Discussion}

Consistent with the existing literature (Borowsky, Ireland \& Resnick, 2001), the adolescents in our sample were about 15 years of age at the time of their attempts. As it has been characterized (Garfinkel et al., 1982), they attempted suicide using means that were available in their homes. While other studies have found drug overdoses to be the most common method to attempt suicide among adolescents (Spirito et al., 1989), cutting was the predominant attempting method in our sample. The majority of the adolescents in our sample chose low-lethality methods (e.g., superficial cutting), although slightly more than half stated that they had intended to end their lives. Adding to this paradox, a few of the participants who stated not having any suicidal intent used highly lethal means (e.g., an overdose with more that 70 Tylenols and severe self-cutting). Many girls denied or minimized their suicidal intent, a finding that is consistent with the literature (Wagner et al., 2002). This leads us to wonder if cognitive distortions - that may be developmentally appropriate-affected the expectations that the girls had about their action's outcomes, or if they became confused while trying to disentangle the rationale for their self-harming behaviors.

These findings highlight the challenges encountered by clinicians when trying to distinguish suicidal behaviors low in lethality from other self-harming behaviors (Wagner et al., 2002). This is 
particularly significant for mental health providers serving teenage girls, because suicide attempts of low lethality are very common within this population (Brent, 1987), and because adolescents often recant their statements of a suicide attempt to avoid hospitalization or the fallout within their families and social networks.

We found the adolescents' self-described behavioral profiles to be of concern. Slightly over two thirds of the participants were within this scale's internalizing and externalizing borderlineclinical or clinical range. Interesting contrasts emerged when we compared these self-reported values with the psychiatric diagnosis given to the attempters. For instance, and consistent with the literature (Jacobson et al., 2008; Kelly, Cornelius, \& Lynch, 2002), the clinicians diagnosed mood disorders for the majority of attempters. However, the number of mood disorders diagnosed by the clinicians was almost twice as high as the the number of internalizing behaviors that the girls reported in the research questionnaires. This may result from the fact that clinicians diagnose when criteria are fully met, which would exclude those subjects reporting borderline-clinical-range profiles. When the girls described their internalizing behaviors, somatic complaint scores were higher than those of withdrawn depressive and anxious depressive behaviors. The clinicians, however, did not seem to capture these somatic complaints as part of the girl's psychiatric diagnosis, as observed by the few medical concerns listed in the Axis III psychiatric diagnosis. Finally, clinicians and adolescents did agree when it came to the girls' anxiety levels.

There is an even greater contrast between the adolescents' reports of externalizing behaviors and the clinical appreciation of the girls' impulse-control issues. The adolescents saw themselves seven times more impulsive and oppositional than the clinicians assessed them to be. The girls' assessment of their impulsivity is in tune with the description of the impulse-control issues common to female suicide attempters whose suicide attempts are low in lethality (Baca-Garcia et al., 200840).

The clinicians did capture the family and environmental stressors associated with Latina adolescents' suicide attempts (Zayas \& Pilat, 2008). This may provide further evidence to the hypothesis that the girls' suicide attempts emerge from conflicts between Latino cultural values and developmentally appropriate autonomy and individuation issues (Zayas et al., 2005). Furthermore, even when almost one quarter of the study participants were born overseas, and all of them were of Latino background, none of the clinicians listed acculturation problems as a focus for treatment. In addition, the clinicians neglected to include adolescents' lifetime attempts in the medical records. This is of particular concern, as previous suicide attempts are common among Latina adolescents (CDC, 2010), and this phenomenon is one of the key predictors of completed suicide (Moscicki, 1999). Finally, the clinicians did not consider the suicide attempt to be a serious symptom, nor did they find these adolescents to be severely mentally ill, as they described symptoms and clinical presentations of the vast majority of adolescents as "moderate" in the DSM-IV-TR's Axis V assessments.

Although the YSR was designed to match behaviorally based clinical syndromes (Achenbach, 1991), previous research comparing self-reports and diagnostic formulations has been inconclusive (Rosenblatt \& Rosenblatt, 2002). In our study, the discrepancies observed between the psychiatric diagnosis rates and the adolescents' self-reported behavioral profiles suggest that we need more studies about how Latina adolescent suicide attempters perceive their own behaviors and how these are perceived by mental health professionals. First, concerning adolescents and drawing from the literature on suicide attempters (Boergers, Spirito, \& Donaldson, 1998), research should focus on whether the girls' pessimistic overview tints the way in which they assess their behaviors. On the clinicians' side, research should assess if the differences between adolescents' self-reports and psychiatric diagnoses result, for instance, from mental health training biases that lead clinicians to see suicide attempts as emerging only from mood disorders and not from impulse-control problems. This is not an issue exclusive to clinical settings, but also seems to permeate measurement tools. For example, the Youth Risk Behavior Surveillance Survey (CDC, 2010) is structured in a manner such that only those subjects answering "yes" to questions related to mood symptoms are led to questions inquiring about suicide ideation and attempts. Lastly, more needs to be understood about the effect that the clinicians' gender and ethnicity may have on the quality of their psychiatric assessments of Latina adolescent suicide attempters. The literature has shown that the ethnic mismatch between mental health providers and clients has a negative effect on the quality of psychiatric services (Zayas, Cabassa, Perez, \& Howard, 2005).

The relationship between suicide and substance-use disorders among young Latinas was of particular interest in this study, as it has been shown that Hispanic females are at significant risk of drug use (Luncheon, Bae, Gonzalez, Lurie, \& Singh, 2008). Furthermore, substance-use disorders place adolescents at risk for attempting suicide (Kelly et al., 2002). Surprisingly, only one participant in our sample had a substance-abuse-related disorder diagnosis. This could be explained by the fact that the average onset of substance abuse-related disorders for Latinas is age 20 (Kessler et al., 2005). Thus, for Latina adolescents, the onset of substance abuse may be subsequent to the establishment of suicidal behavioral patterns.

Confirming what the CDC (2010) has described, the majority of the adolescents included in this study had a lifetime history of two to more than six previous suicide attempts. We do not know if the girls received any medical or mental health services following those earlier attempts, when those attempts took place, or what their lethality was. This study collected cross-sectional data, and thus we do not know the suicidal trajectories of the enrolled girls after they completed their participation. The literature has described that adolescent suicide attempters commonly reattempt within the 3-6 months following the first incidence, but also that a first attempt's predictive power of more attempts extends for at least 12 years (Bridge, Goldstein, \& Brent, 2006). This finding highlights the need to conduct longitudinal research projects following Latina suicide attempters, as a means to test the suicidal trajectories of this population, as well as to quantify the taxing effects these behaviors have on the health care system.

In tune with existing literature (Borowsky et al., 2001) the adolescents studied here attempted suicide at an early age. Combining these data with the lifetime history of attempts and with the likelihood of future incidents, it becomes clear that this group of young females presents unique vulnerabilities. These vulnerabilities may be connected with unidentified developmental risks for Latina adolescents growing up in the U.S. that need further exploration. 


\section{Limitations}

We are mindful of several limitations to this study. First, our findings come from a small sample size and participants were not randomly selected. The purpose of the study, however, was not to make complex statistical comparisons, but to describe themes emerging from the adolescents' medical charts, their self-reported behavioral profiles in the questionnaires, and their stories as shared with us in the qualitative interviews. Second, not all subjects in the sample completed a qualitative interview, limiting our ability to analyze their intent even further. Third, the medical charts were the sole data source for the adolescents' psychiatric diagnostics. Psychiatric diagnoses obtained from medical charts have been shown to be unreliable (Gilbert, Lowenstein, Koziol-McLain, Barta, \& Steiner, 1996). In addition, we lack information about the demographic profiles and training of the professionals that diagnosed the adolescents, or about the collateral data used to inform their diagnostic formulations. These two limitations make it difficult to determine if the discrepancies found between the teen's selfreported behavioral profiles and their diagnoses would replicate in other settings. Fourth and finally, the label of "suicide attempter" given to each of the girls in this sample was based on clinical assessments recorded on medical charts. We know nothing about the rationale informing this labeling, or about other data that may have been accessed by clinicians to justify their professional judgments.

\section{Recommendations and Implications}

Latina adolescents carry a great risk for suicide attempts. Findings from this study indicate that mental health professionals should explore both internalizing and externalizing behaviors as pathways for suicide attempts among these patients. Further consideration needs to be given to the exploration of somatic complaints and to the assessment of acculturation problems and lifetime attempts when conducting psychiatric assessments of and defining treatment approaches for Latina adolescent suicide attempters. These findings highlight the importance of future research on the self-described behavioral profiles of Latina adolescent suicide attempters and on the clinical assessment of these patients.

\section{References}

Achenbach, T. M. (1991). Manual for the Child Behavior Checklist/4-18. Burlington, VT: University of Vermont Department of Psychiatry. American Psychiatric Association. (2000). Diagnostic and statistical manual of mental disorders (4th ed.). Washington, DC: Author.

Baca-Garcia, E., Diaz-Sastre, C., Garc'ia Resa, E., Blasco, H., Braquehais Conesa, D., Oquendo, M. A., ... de Leon, J. (2005). Suicide attempts and impulsivity. European Archives of Psychiatry and Clinical Neuroscience, 255, 152-156, doi:10.1007/s00406-004-0549-3

Berman, A. L., Shepherd, G., \& Silverman, M. M. (2003). The LSARS-II: Lethality of Suicide Attempt Rating Scale-updated. Suicide and LifeThreatening Behavior, 33, 261-276. doi:10.1521/suli.33.3.261.23211

Blasco-Fontecilla, H., Baca-Garcia, E., Dervic, K., Perez-Rodriguez, M. M., Saiz-Gonzalez, M. D., Saiz-Ruiz, J., ... De Leon, J. (2009). Severity of personality disorders and suicide attempt. Acta Psychiatrica Scandinavica, 119, 149-155. doi:10.1111/j.1600-0447.2008.01284.x

Boergers, J., Spirito, A., \& Donaldson, D. (1998). Reasons for adolescent suicide attempts: Associations with psychological functioning. Journal of the American Academy of Child \& Adolescent Psychiatry, 37, 12871293. doi:10.1097/00004583-199812000-00012

Borowsky, I. W., Ireland, M., \& Resnick, M. D. (2001). Adolescent suicide attempts: Risks and protectors. Pediatrics, 107, 485-493. doi:10.1542/ peds.107.3.485

Brent, D. A. (1987). Correlates of the medical lethality of suicide attempts in children and adolescents. Journal of the American Academy of Child \& Adolescent Psychiatry, 26, 87-91. doi:10.1097/00004583198701000-00017

Bridge, J. A., Goldstein, T. R., \& Brent, D. A. (2006). Adolescent suicide and suicidal behavior. Journal of Child Psychology and Psychiatry, 47, 372-394. doi:10.1111/j.1469-7610.2006.01615.x

Brown, G. K., Henriques, G. R., Sosdjan, D., \& Beck, A. T. (2004). Suicide intent and accurate expectations of lethality: Predictors of medical lethality of suicide attempts. Journal of Consulting and Clinical Psychology, 72, 1170-1174. doi:10.1037/0022-006X.72.6.1170

Centers for Disease Control and Prevention. (2010). Youth risk behavior surveillance: United States, 2009. Morbidity and Mortality Weekly Report: Surveillance Summaries, 59(SS-5).

Garcia, C., Skay, C., Sieving, R., Naughton, S., \& Bearinger, L. H. (2008). Family and racial factors associated with suicide and emotional distress among Latino students. Journal of School Health, 78, 487-495. doi: 10.1111/j.1746-1561.2008.00334.x

Garfinkel, B. D., Froese, A., \& Hood, J. (1982). Suicide attempts in children and adolescents. The American Journal of Psychiatry, 139, $1257-1261$

Gilbert, E. H., Lowenstein, S. R., Koziol-McLain, J., Barta, D. C., \& Steiner, J. (1996). Chart reviews in emergency medicine research: Where are the methods? Annals of Emergency Medicine, 27, 305-308. doi:10.1016/S0196-0644(96)70264-0

Goldsmith, S. K., Pellmar, T. C., Kleinman, A. M., \& Bunney, W. E. (2002). Reducing suicide: A national imperative. Washington, DC: National Academy Press.

Harris, H. E., \& Myers, W. C. (1997). Adolescents' misperceptions of the dangerousness of acetaminophen overdose. Suicide and LifeThreatening Behavior, 27, 274-277.

Jacobson, C. M., Muehlenkamp, J. J., Miller, A. L., \& Turner, J. B. (2008). Psychiatric impairment among adolescents engaging in different types of deliberate self-harm. Journal of Clinical Child and Adolescent Psychology, 37, 363-375. doi:10.1080/15374410801955771

Kelly, T. M., Cornelius, J. R., \& Lynch, K. G. (2002). Psychiatric and substance use disorders as risk factors for attempted suicide among adolescents: A case control study. Suicide and Life-Threatening Behavior, 32, 301-312. doi:10.1521/suli.32.3.301.22168

Kessler, R. C., Berglund, P., Demler, O., Jin, R., Merikangas, K. R., \& Walters, E. E. (2005). Lifetime prevalence and age-of-onset distributions of DSM-IV disorders in the national comorbidity survey replication. Archives of General Psychiatry, 62, 593-602. doi:10.1001/archpsyc.62.6.593

Luncheon, C., Bae, S., Gonzalez, A., Lurie, S., \& Singh, K. P. (2008). Hispanic female adolescents' use of illicit drugs and the risk of suicidal thoughts. American Journal of Health Behavior, 32, 52-59.

Maxwell, J. A. (1992). Understanding and validity in qualitative research. Harvard Educational Review, 62, 279-300.

Mazza, J. J., \& Reynolds, W. M. (2001). An investigation of psychopathology in nonreferred suicidal and nonsuicidal adolescents. Suicide and Life-Threatening Behavior, 31, 282-302. doi:10.1521/suli.31.3.282 .24245

Moscicki, E. K. (1999). Epidemiology of suicide. In D. J. Jacobs (Ed.), The Harvard Medical School guide to suicide assessment and intervention. Hoboken, NJ: Jossey-Bass.

Muehlenkamp, J. J., \& Gutierrez, P. M. (2004). An investigation of differences between self-injurious behavior and suicide attempts in a 
sample of adolescents. Suicide and Life-Threatening Behavior, 34, 1223. doi:10.1521/suli.34.1.12.27769

Nasser, E. H., \& Overholser, J. C. (1999). Assessing varying degrees of lethality in depressed adolescent suicide attempters. Acta Psychiatrica Scandinavica, 99, 423-431. doi:10.1111/j.1600-0447.1999.tb00988.x

Overholser, J. C., \& Spirito, A. (2003). Precursors to adolescent suicide attempts. In: A. Spirito \& J. C. Overholser (Eds.), Evaluating and treating adolescent suicide attempters: From research to practice (pp. 19-40). San Diego, CA: Elsevier Science. doi:10.1016/B978012657951-2/50003-0

Pew Hispanic Center. (2009). Between two worlds: How young Latinos come of age in America. Washington, DC: Author.

Range, L. M., \& Knott, E. C. (1997). Twenty suicide assessment instruments: Evaluation and recommendations. Death Studies, 21, 25-58. doi:10.1080/074811897202128

Rosenblatt, A., \& Rosenblatt, J. A. (2002). Assessing the effectiveness of care for youth with severe emotional disturbances. Is there agreement between popular outcome measures? Journal of Behavioral Health Services \& Research, 29, 259-273. doi:10.1007/BF02287367

Silverman, M. M., Berman, A. L., Sandal, N. D., O'Carroll, P. W., \& Joiner, T. E. (2007). Rebuilding the Tower of Babel: A revised nomenclature for the study of suicide and suicidal behaviors Part I: Background, rationale. Suicide and Life-Threatening Behavior, 37, 245-247.

Smith, K., Conroy, R., \& Ehler, B. (1984). Lethality of Suicide Attempt Rating Scale. Suicide and Life-Threatening Behavior, 14, 215-242.

Spirito, A., Overholser, J. C., \& Stark, L. (1989). Common problems and coping strategies, II: Findings with adolescent suicide attempters. Journal of Personality Assessment, 67, 90-101. doi:10.1207/ s15327752jpa6701_7

Spirito, A., \& Overholser, J. C. (2003). The suicidal child: Assessment and management of adolescents after a suicide attempt. Child and Adolescent Psychiatric Clinics of North America, 12, 649-665. doi:10.1016/S10564993(03)00034-8
Turner, S. G., Kaplan, C. P., Zayas, L. H., \& Ross, R. E. (2002). Suicide attempts by adolescent Latinas: An exploratory study of individual and family correlates. Child and Adolescent Social Work Journal, 19, 357374. doi:10.1023/A:1020270430436

Velting, D. M., Rathus, J. H., \& Asnis, G. M. (1998). Asking adolescents to explain discrepancies in self-reported suicidality. Suicide and LifeThreatening Behavior, 28, 187-196.

Wagner, B. M., Wong, S. A., \& Jobes, D. A. (2002). Mental health professionals' determinations of adolescent suicide attempts. Suicide and Life-Threatening Behavior, 32, 284-300. doi:10.1521/ suli.32.3.284.22178

Zayas, L. H., Cabassa, L. J., Perez, M. C., \& Howard, M. O. (2005). Language and ethnicity are parts of the interpersonal process that converge during psychiatric diagnosis, affecting diagnostic accuracy and concordance among clinicians. Journal of Ethnic \& Cultural Diversity in Social Work: Innovation in Theory, Research \& Practice, 14, 93-109. doi:10.1300/J051v14n01_05

Zayas, L. H., Gulbas, L. E., Fedoravicius, N., \& Cabassa, L. J. (2010). Patterns of distress, precipitating events, and reflections on suicide attempts by young Latinas. Social Science \& Medicine, 70, 1773-1779. doi:10.1016/j.socscimed.2010.02.013

Zayas, L. H., Lester, R., Cabassa, L. J., \& Fortuna, L. (2005). Why do so many Latina teens attempt suicide? A conceptual model for research. American Journal of Orthopsychiatry, 75, 275-287. doi:10.1037/00029432.75.2.275

Zayas, L. H., \& Pilat, A. M. (2008). Suicidal behavior in Latinas: Explanatory cultural factors and implications for intervention. Suicide and Life Threatening Behavior, 38, 334-342. doi:10.1521/suli.2008.38.3.334

Received March 28, 2011

Revision received September 30, 2011

Accepted October 3, 2011 